\title{
Original Article (short paper) \\ Exercise training reverse autonomic dysfunction and hypertension in rats fed with high-fat diet
}

\author{
Carla Cristina de Sordi ${ }^{1}$, Marina de Paiva Lemos ${ }^{1}$, Moacir Marocolo² , Rosangela Soares Chriguer ${ }^{3}$, Edmar Lacerda \\ Mendes $^{1}$, Gustavo Ribeiro da Mota ${ }^{1}$, Octávio Barbosa Neto ${ }^{1}$ \\ ${ }^{1}$ Universidade Federal do Triângulo Mineiro, UFTM, Uberada, MG, Brazil; ${ }^{2}$ Universidade Federal de Juiz de \\ Fora, UFJF, Juiz de Fora, MG, Brazil, ${ }^{3}$ Universidade Federal de São Paulo, UNIFESP, Santos, SP, Brazil.
}

\begin{abstract}
Aims: We evaluated whether exercise training ameliorates cardiovascular autonomic dysfunction in obesity-induced by high-fat diet (HFD) in rats. Methods: Wistar male rats were assigned in normal diet, sedentary (ND-S) and trained (ND-T) and HFD, sedentary (HFD-S) and trained (HFD-T). Blood pressure (BP), heart rate (HR), HR variability (HRV), BP variability (BPV), cardiac baroreflex and cardiac autonomic tonus were assessed. Results: HFD-S showed higher bodyweight increase (19.4\%) compared to all other groups (HFD-T: $13.2 \%$, ND-S: $14 \%$ and ND-T: $12.4 \%$ ). Relative epididymal, retroperitoneal and visceral fat was also greater in HFD-S compared to all other groups. Resting bradycardia in ND-T $(339.5 \pm 10.6 \mathrm{bpm})$ and HFD-T $(341.0 \pm 9.4 \mathrm{bpm})$ was more pronounced than ND-S $(438.4 \pm 6.3 \mathrm{bpm} ; \mathrm{p}<0.05)$ and HFD-S $(448.5 \pm 18.7 \mathrm{bpm}$; $\mathrm{p}<0.05)$. The HFD-T group showed lower systolic $(125.3 \pm 1.9 \mathrm{mmHg})$, diastolic $(88.5 \pm 2.0 \mathrm{mmHg})$ and mean BP $(100.3 \pm 2.5 \mathrm{mmHg})$ in comparison with HFD-S $(153.8 \pm 3.7 ; 103.5 \pm 2.6$ and $120.5 \pm 3.7 \mathrm{mmHg} ; \mathrm{p}<0.05$, respectively). Lower variance of $\mathrm{HRV}$ and higher variance of diastolic BPV was observed in HFD-S compared to other groups while sympathetic modulation of HRV and BPV was higher in HFD-S, as well as the LF/HF ratio. HFD-T showed a decrease in sympathetic tonus compared to HFD-S. ND-T and HFD-T showed higher cardiac vagal tonus than respective sedentary groups. Analysis showed an association between visceral fat, sympathetic activity and BP. Conclusions: HFD induces hypertension and is associated with autonomic imbalance in rats, while exercise training may reverse these harmful changes.
\end{abstract}

Keywords: exercise training, high fat diet, obesity, cardiovascular autonomic dysfunction.

\section{Introduction}

Contemporary lifestyle is often characterized by sedentary behavior and an excess intake of a high-fat diet (HFD), increasing the chances of developing of obesity ${ }^{1}$ and several chronic diseases, including arterial hypertension ${ }^{2}$ and autonomic alterations ${ }^{3}$. Conflicting results have emerged over the nature of autonomic activity in obesity ${ }^{4}$. These investigators showed that obese subjects presented lower sympathetic activity but no change in parasympathetic modulation. On the contrary, an increase in body weight (BW) is associated with sympathetic overactivity and attenuation in parasympathetic activity ${ }^{5}$. Likewise, obese subjects have shown an imbalance in autonomic nervous system (ANS) activity, which is characterized by an increase in parasympathetic nervous system (PNS) tonus and/or a decrease in the sympathetic nervous system (SNS) tonus'.

Obesity was found to be associated with decreased sympathetic activity in obesity ${ }^{7}$. Others researcher's, however, also reports a sympathetic hyperactivity ${ }^{2}$. In animal models, HFD-induced obesity increases sympathetic activity and renin-angiotensin-aldosterone system, consequently increasing blood pressure (BP $)^{8}$. Obesity not only shifts SNS, but it also changes cardiac PNS drive. The HFD has been found to reduce parasympathetic activity in rats ${ }^{9}$. Body mass index (BMI), waist circumference and sum of skinfolds are associated with a reduced cardiac vagal tonus and indicators of central obesity as compared to total indicators of obesity ${ }^{10}$. Increase of $\mathrm{BW}$ is associated with a decline in PNS, accompanied by a rise in heart rate (HR), and conversely, HR declines during weight reduction This is a point important because increment in HR has been shown as a marker associated with increased mortality ${ }^{11}$.
Exercise training ameliorates BP levels in overweight and lean subjects, as well as was effective in reducing sympathetic and parasympathetic nervous activities in obesity ${ }^{12}$. Chronic exercise may prevent obesity development and metabolic alteration ${ }^{13}$. Despite much study in this field, the mechanisms through which excessive fat accumulation results in an increased incidence of cardiovascular autonomic dysfunction and the possible effects of ET in these parameters are still a matter of debate. The mechanisms underlying this relationship are multifactorial, and for some time there are controversy as to whether the SNS and PNS were activated or inhibited in obesity and the effects of ET. Therefore, we determined the alteration of baseline pressoric regulation, autonomic nerve activity by HR and BP variability, cardiac autonomic tonus and baroreflex sensitivity in HFD-fed rats after swimming exercise training. This study aimed to clarify the therapeutic effect of exercise training on sympathetic activation and parasympathetic attenuation as well as bradycardic and tachycardic baroreflex responses in diet-induced obese rats.

\section{Materials and methods}

\section{Animal model}

Adult male Wistar rats at 10-12 weeks of age and BW around $380-400 \mathrm{~g}$ were procured from Physiology Laboratory. The animals were kept at a constant temperature of $22 \pm 1{ }^{\circ} \mathrm{C}$, relative humidity of $50-60 \%$ and light/dark cycle (12/12 h). They were fed with different diets as described below and drinking water ad libitum. The rats were randomly assigned to 4 experimental 
groups that received either a normal diet plus sedentary activity (ND-S, $n=8$ ), a high-fat diet plus sedentary activity (HFD-S, $n=8)$, a normal diet plus exercise training activity (ND-T, $n=8)$ and a high-fat diet plus exercise training activity (HFD-T, $n=8)$. All experimental procedures were performed in accordance with the Ethical Principles in Animal Research of the Brazilian College of Animal Experimentation and were approved by the local Ethical Committee for Animal Research (Protocol 167/2010).

\section{Diet and exercise training}

A 9-week dietary intervention was followed by a same period of exercise intervention. The both ND groups received a standard diet, normocaloric (commercial Nuvilab ${ }^{\circledR}$ CR1 rat feeding) containing (\% weight) $19 \%$ protein, $56 \%$ carbohydrate, $3.5 \%$ lipids, $4.5 \%$ cellulose and $5 \%$ vitamins and minerals, while the HFD groups received a diet consisted of a mixture of ground and calorie foods that were offered in the form of pellets. They were fed with (\% weight) $15 \mathrm{~g}$ of standard diet (Nuvilab ${ }^{\circledR} \mathrm{CR} 1$ ), $10 \mathrm{~g}$ roasted peanuts, $10 \mathrm{~g}$ milk chocolate and $5 \mathrm{~g}$ of cornstarch cookie. This type of diet had in its composition $20 \%$ protein, $48 \%$ carbohydrate, $20 \%$ as fat, $4 \%$ cellulose and $5 \%$ of vitamins and minerals ${ }^{14}$. The swimming protocol was performed in a glass tank and ambient water temperature was kept at $30^{\circ} \pm 1^{\circ} \mathrm{C}$. The trained animals received a 20 min adaptation period on the first day and were increased 10-min each day until reaching 1 $\mathrm{h}$ on the fifty day ${ }^{15}$. Following, these rats trained 5 days/week with a gradual progression toward a $2 \mathrm{~h}$ session. This protocol is defined as an aerobic endurance training and low-intensity, as the animals swam without additional work load, this method corresponds the intensity below the anaerobic threshold in rats ${ }^{16}$. Sedentary animals were placed in the swimming apparatus for 10 min twice a week to mimic the water stress associated with the experimental protocol. BW was measured in a conscious state at weekly intervals.

\section{Experimental procedures and hemodynamic recording protocol}

One day after the last exercise session, acute experiments were performed under anesthesia induced by intraperitoneal injection of sodium pentobarbital $(40 \mathrm{mg} / \mathrm{kg})$. Arterial and venous catheters (PE-10 attached to PE-50) filled with heparinized saline were inserted in the femoral artery and vein to direct recordings of pulsatile BP and drug administration, respectively. Followed, the catheters were tunneled subcutaneously and exteriorized at the scapular region. Twenty-four hours after catheter surgery completion, BP and HR were monitored in conscious freely moving rats by connecting the arterial catheter to a pressure transducer $(\mathrm{P} 23 \mathrm{Db}$, Gould-Statham) connected to amplifier (ML224 Quad Bridge Amp, ADInstruments, NSW, Australia) and a microcomputer equipped with an analog-to-digital converter board (CODAS, 4-kHz sampling frequency, Di220 Dataq Instruments, Inc., Akron, $\mathrm{OH}, \mathrm{USA}$ ). After animal acclimation to the experimental room, the hemodynamic parameters were recorded for $30 \mathrm{~min}$. Mean
(MBP), systolic (SBP), and diastolic BP (DBP) were analyzed off-line. Beat-by-beat pulse interval (PI) values were generated off-line from the pulsatile arterial BP signal by measuring the time interval between two systolic peaks.

\section{Linear analysis of $H R$ and $B P$ variability}

For the cardiovascular variability study, the signals of BP were processed to generate beat-to-beat time series of PI, DBP, and SBP. The variance of these values in each period was considered a variability index in the time-domain assessed by the standard deviation of the time series. The PI, DBP and SBP fluctuations were assessed in the frequency domain by means of autoregressive spectral estimation method as described elsewhere ${ }^{17}$. Briefly, beat by beat time series of PI, DBP, and SBP were divided into serial segments of 300 beats with a $50 \%$ overlap on the previous segments (Welch's method). The spectra of each segment were estimated via the Levinson-Durbin recursion, and the order of the model chosen according to Akaike's criterion ${ }^{18}$. The oscillatory components were labeled as very low (VLF, 0.01 to $0.20 \mathrm{~Hz}$ ), low (LF, 0.20 to $0.75 \mathrm{~Hz}$ ) or high frequency (HF, 0.75 to $3.00 \mathrm{~Hz}$ ). The normalization procedure, applied only to the variability of the PI, was performed by dividing the power of the LF or HF component by total spectral power, which is subtracted from the power of the VLF band and multiplying the result by $100^{19}$. The normalization procedure tends to minimize the effect of the changes in total power on the absolute values of LF and HF components of HRV ${ }^{17}$.

\section{Baroreflex sensitivity and cardiac autonomic tonus}

In order to assess the baroreflex sensitivity, we administered intravenous injections of phenylephrine $\left(2 \mu \mathrm{g} \cdot \mathrm{kg}^{-1}\right)$ and sodium nitroprusside $\left(2 \mu \mathrm{g} \cdot \mathrm{kg}^{-1}\right)$ to elicit alterations in BP, which was evaluated by the slope of the regression line obtained by best-fit points relating changes in HR and MBP. A 5-minute interval between doses was necessary for BP to return to baseline. Subsequently, we also performed the sympathetic and vagal autonomic blockade after propranolol ( $5 \mathrm{mg} / \mathrm{kg}$, i.v. $)$ and atropine ( $4 \mathrm{mg} / \mathrm{kg}$, i.v.) injections, respectively, to calculate the sympathetic and vagal tonus, as well as the pacemarker intrinsic HR (IHR) and tonic sympathovagal index. The autonomic blockers were administered in a random sequence with a 15 min interval between them. After double blockade, the cardiovascular recordings lasted for $15 \mathrm{~min}$. Briefly, vagal tonus was estimated by the difference between the maximal bradycardia achieved after sympathetic blockade with propranolol (double blockade by atropine and propranolol) and the pacemarker IHR. Sympathetic tonus was evaluated by the difference between the highest tachycardia observed after vagal blockade with atropine and the pacemarker IHR. The tonic sympathovagal index was obtained as the ratio between resting and intrinsic $\mathrm{HR}$. 


\section{Removal of white adipose tissue depot.}

At the completion of the experiment, rats were euthanized with an overdose of pentobarbital sodium $(300 \mathrm{mg} / \mathrm{kg})$. The epididymal, retroperitoneal (surrounding the kidney) and visceral (surrounding the stomach and intestines) white adipose tissue were rapidly removed, rinsed in deionized water to remove excess blood and weighed.

\section{Statistical analysis}

The normality of the data was checked with the KolmogorovSmirnov test and Levene's F test was used to assess the homogeneity. Significance of differences of continuous variables between normal-fed and high fat-diet animals and exercise training was tested using 2-way ANOVA with Tukey post-test (normally distributed variables). Non-normally distributed variables were tested by non-parametric Friedman test followed by Mann-Whitney U-tests. Pearson correlation coefficient was used to test the correlation between white adipose tissue with sympathetic activity and blood pressure levels. All data are represented as mean \pm SEM. Probability values of $p<0.05$ were considered statistically significant. Analyses were performed using SigmaStat ${ }^{\circledR}$ SPSS, Chicago, IL.

\section{Results}

HFD intake induced obesity in sedentary rats as evidenced by the increased final BW, BW gain and adiposity (masses of epididymal, retroperitoneal and visceral adipose depots) in comparison to the other groups $(\mathrm{p}<0.05)$. The ET was able to prevent the increases in the above abnormal anatomic data in HFD-induced obese rats $(\mathrm{p}<0.05)$, similarly to sedentary and trained animals with normal diet (Table 1).

Table 1. Descriptive data for final BW mass, BW gain (relative D\%), absolute and relative weights of epididymal, retroperitoneal and visceral adipose tissue weights from sedentary normal diet (ND-S, $n=8)$, sedentary high-fat diet (HFD-S, n=8), trained normal diet (ND-T, $n=8)$ and trained high-fat diet (HFD-T, $\mathrm{n}=8$ ) groups for 9 weeks.

\begin{tabular}{|c|c|c|c|c|}
\hline & ND-S & HFD-S & ND-T & HFD-T \\
\hline Initial BW (g) & $390.7 \pm 13.1$ & $392.4 \pm 9.1$ & $391.6 \pm 6.3$ & $393.3 \pm 7.3$ \\
\hline Final BW (g) & $445.6 \pm 3.9$ & $467.2 \pm 5.0^{*}$ & $440.4 \pm 4.3^{\dagger}$ & $446.1 \pm 3.5^{\dagger}$ \\
\hline BW gain, $(\Delta \%)$ & $14.0 \pm 1.7$ & $19.6 \pm 2,1^{*}$ & $12.4 \pm 6.1^{\dagger}$ & $13.2 \pm 4.4^{\dagger}$ \\
\hline Epi WAT (g) & $0.81 \pm 0.03$ & $1.64 \pm 0.05^{*}$ & $0.65 \pm 0.05^{\dagger}$ & $0.79 \pm 0.03^{\dagger+}$ \\
\hline Rel. epi WAT (\%) & $0.18 \pm 0.3$ & $0.34 \pm 0.4^{*}$ & $0.13 \pm 0.3^{\dagger}$ & $0.17 \pm 0.2^{\dagger \ddagger}$ \\
\hline Retro WAT (g) & $1.26 \pm 0.05$ & $3.38 \pm 0.14^{*}$ & $1.11 \pm 0.06^{\dagger}$ & $2.23 \pm 0.11^{* *}$ \\
\hline Rel. retro WAT (\%) & $0.23 \pm 0.1$ & $0.65 \pm 0.4^{*}$ & $0.02 \pm 0.2^{\dagger}$ & $0.45 \pm 0.3$ \\
\hline Visc WAT (g) & $1.05 \pm 0.04$ & $1.91 \pm 0.02^{*}$ & $0.75 \pm 0.07^{\dagger}$ & $0.98 \pm 0.07^{\text {t* }}$ \\
\hline Rel. visc WAT (\%) & $0.22 \pm 0.2$ & $0.40 \pm 0.3^{*}$ & $0.16 \pm 0.4^{\dagger}$ & $0.20 \pm 0.2^{\dagger}$ \\
\hline
\end{tabular}

Values are mean \pm SEM. Abbreviations: BW; body weight, WAT; white adipose tissue, Epi; epididymal, Retro; retroperitoneal, Visc; visceral. Significances are ${ }^{*} \mathrm{p}<0.05$ vs. ND-S; $\uparrow \mathrm{p}<0.05$ vs. HFD-S and $\$ \mathrm{p}<0.05$ vs. ND-T. (Statistical differences were determined by 2 -way ANOVA followed by Tukey post-test).

Under baseline conditions, resting bradycardia in both ND-T $(339.5 \pm 10.6 \mathrm{bpm})$ and HFD-T $(341.0 \pm 9.4 \mathrm{bpm})$ male rats has been more closely pronounced when compared with their respective sedentary groups $(438.4 \pm 6.3 \mathrm{bpm} \mathrm{in} \mathrm{ND-S} \mathrm{and} 448.5$ \pm 18.7 bpm in HFD-S, $p<0.05$; Fig. 1 A). The sedentary rats fed a HFD exhibited a higher systolic BP $(153.8 \pm 3.7 \mathrm{mmHg})$ when compared with HFD-T $(125.3 \pm 1.9 \mathrm{mmHg} ; \mathrm{p}<0.001)$, ND-S
$(123.9 \pm 3.9 \mathrm{mmHg} ; \mathrm{p}<0.001)$ and ND-T $(121.8 \pm 5.4 \mathrm{mmHg}$, $\mathrm{p}<0.001$; Fig. 1B). Similar results were observed for diastolic BP in HFD-S $(103.5 \pm 2.6 \mathrm{mmHg})$ versus HFD-T $(88.5 \pm 2.0$ $\mathrm{mmHg}$; $<0.05)$, ND-S $(87.4 \pm 4.2 \mathrm{mmHg} ; \mathrm{p}<0.05)$ and ND-T $(86.7 \pm 2.8 \mathrm{mmHg}, \mathrm{p}<0.05$; Fig. 1C). Consequently, the mean BP was higher in HFD-S $(120.5 \pm 3.7 \mathrm{mmHg})$ than in HFD-T $(100.3$ $\pm 2.5 \mathrm{mmHg} ; \mathrm{p}<0.001)$, ND-S $(99.6 \pm 5.1 \mathrm{mmHg} ; \mathrm{p}<0.001)$ and 
ND-T $(98.4 \pm 4.1 \mathrm{mmHg}$; $<<0.001)$, respectively (Fig. 1D). No differences were observed in BP between sedentary and trained rats fed a normal diet.

The variance of HRV was lower in HFD-S when compared withND-S, ND-TandHFD-Tanimals $(\mathrm{p}<0.05)$. On the otherhand, the variance of DBPV increased in comparison to the same groups $(p<0.05)$. The LF component of HRV, SBPV and DBPV were higher in HFD-S than in the other groups $(p<0.05)$. The HF power of HRV was lower in sedentary rats submitted to HFD compared to ND-S, ND-T and HFD-T $(\mathrm{p}<0.05)$. Considered a hallmark of sympatho-vagal balance, the LF/HF relation was higher in HFD-S rats in comparison to the other groups $(\mathrm{p}<0.05)$ (Table 2$)$.

Figure 1. Values of (a) resting heart rate (HR, bpm), (b) systolic blood pressure (SBP, mmHg), (c) diastolic blood pressure (DBP, mmHg) and (d) mean blood pressure (MBP, $\mathrm{mmHg}$ ) in non-anesthetized Wistar rats submitted to normal diet plus sedentary activity (ND-S, $\mathrm{n}=8$ ), high-fat diet plus sedentary activity (HFD-S, $n=8$ ), normal diet plus exercise training activity (ND-T, $n=8$ ) and high-fat diet plus exercise training activity (HFD-T, $\mathrm{n}=8$ ) treatment during 9 weeks. Bars represent mean \pm SEM. ${ }^{*} \mathrm{p}<0.05$ vs. ND-S, $\uparrow \mathrm{p}<0.05$ vs. HFD-S, $\$ \mathrm{p}<0.05$ vs. ND-T. (Statistical differences were determined by 2-way ANOVA followed by Tukey post-test).
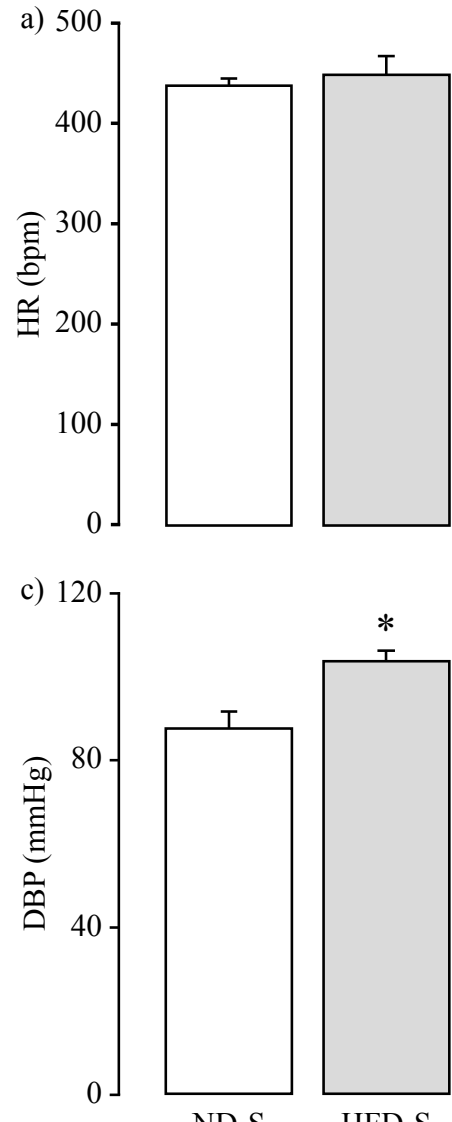

ND-S

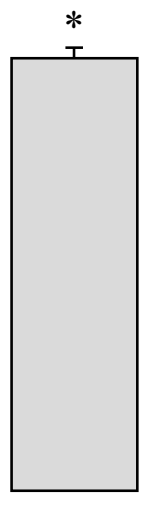

HFD-S
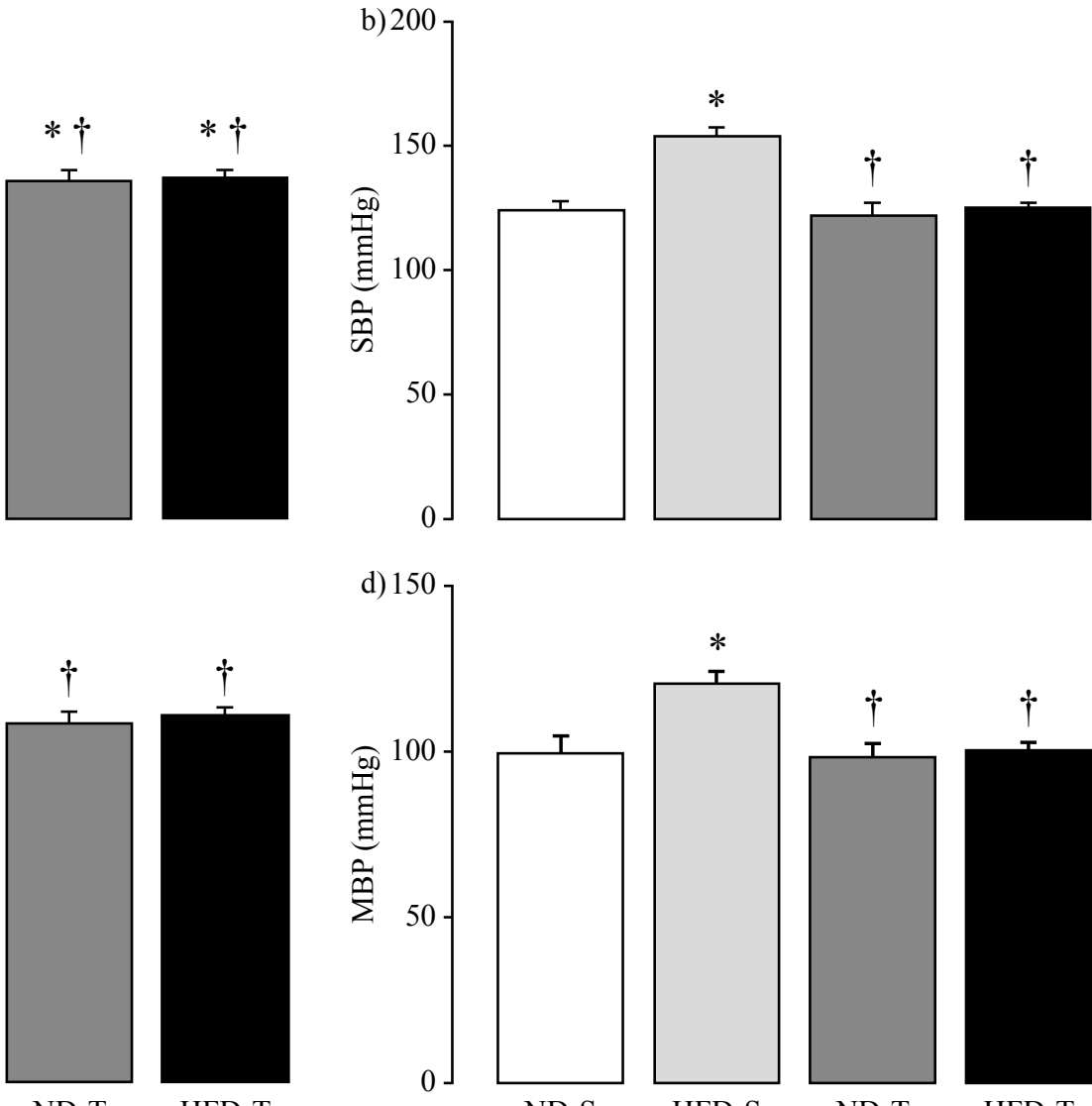

HFD-T

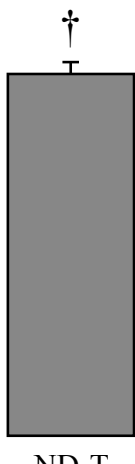

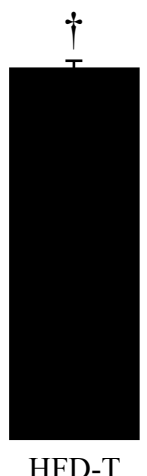
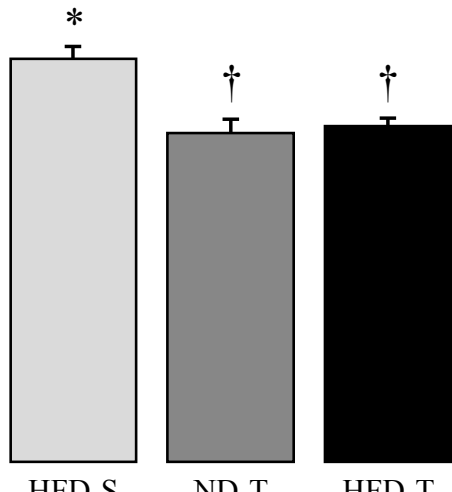

HFD-T

Table 2. Mean values ( \pm SEM) of variance and VLF, LF, and HF spectral components of systolic and diastolic blood pressure variability and pulse interval variability in non-anesthetized Wistar rats submitted to normal diet plus sedentary activity (ND-S, n=8), high-fat diet plus sedentary activity (HFD-S, $n=8$ ), normal diet plus exercise training activity (ND-T, $n=8$ ) and high-fat diet plus exercise training activity (HFD-T, $n=8$ ) treatment during 9 weeks.

\begin{tabular}{|c|c|c|c|c|}
\hline & ND-S & HFD-S & ND-T & HFD-T \\
\hline \multicolumn{5}{|l|}{ Spectral parameters } \\
\hline \multicolumn{5}{|l|}{ SBP } \\
\hline Variance, $\mathbf{m m H g}^{2}$ & $11.7 \pm 1.8$ & $12.7 \pm 1.7$ & $12.9 \pm 1.5$ & $14.5 \pm 2.4$ \\
\hline $\mathbf{L F}, \mathbf{m m H g}^{2}$ & $5.7 \pm 0.9$ & $12.9 \pm 1.8^{*}$ & $4.2 \pm 1.0^{\dagger}$ & $7.5 \pm 1.7^{\dagger}$ \\
\hline $\mathrm{HF}, \mathrm{mmHg}^{2}$ & $7.3 \pm 0.4$ & $5.9 \pm 3.2$ & $8.6 \pm 1.5$ & $7.9 \pm 0.6$ \\
\hline \multicolumn{5}{|l|}{ DBP } \\
\hline Variance, $\mathbf{m m H g}^{2}$ & $11.2 \pm 0.7$ & $17.0 \pm 1.5^{*}$ & $11.3 \pm 0.9^{\dagger}$ & $12.8 \pm 1.4^{\dagger}$ \\
\hline $\mathbf{L F}, \mathbf{m m H g}^{2}$ & $3.4 \pm 0.4$ & $9.6 \pm 0.5^{*}$ & $3.5 \pm 0.4^{\dagger}$ & $4.7 \pm 0.5^{\dagger}$ \\
\hline $\mathrm{HF}, \mathrm{mmHg}^{2}$ & $3.9 \pm 0.3$ & $3.7 \pm 0.6$ & $3.6 \pm 2.9$ & $4.4 \pm 1.5$ \\
\hline
\end{tabular}




\begin{tabular}{|c|c|c|c|c|}
\hline PI & & & & \\
\hline Variance, $\mathbf{m s}^{2}$ & $27.7 \pm 0.9$ & $19.9 \pm 0.6^{*}$ & $30.6 \pm 1.3^{\dagger}$ & $24.3 \pm 5.4$ \\
\hline VLF, $\mathbf{m s}^{2}$ & $8.3 \pm 1.2$ & $11.4 \pm 1.7$ & $7.4 \pm 1.8$ & $8.9 \pm 1.6$ \\
\hline $\mathbf{L F}, \mathbf{m s}^{2}$ & $4.1 \pm 1.0$ & $10.2 \pm 1.6^{*}$ & $4.2 \pm 1.1^{\dagger}$ & $4.1 \pm 1.8^{\dagger}$ \\
\hline LF, nu & $13.7 \pm 2.5$ & $19.5 \pm 3.9$ & $12.7 \pm 1.5$ & $11.8 \pm 1.6$ \\
\hline $\mathrm{HF}, \mathrm{ms}^{2}$ & $14.7 \pm 0.7$ & $7.7 \pm 0.9^{*}$ & $20.9 \pm 2.2^{\dagger}$ & $18.3 \pm 1.2^{\dagger}$ \\
\hline HF, nu & $74.7 \pm 2.4$ & $70.3 \pm 6.3$ & $77.1 \pm 6.2$ & $83.3 \pm 1.7$ \\
\hline LF/HF & $0.27 \pm 0.0$ & $1.58 \pm 0.3^{*}$ & $0.26 \pm 0.0^{\dagger}$ & $0.23 \pm 0.0^{\dagger}$ \\
\hline
\end{tabular}

Abbreviations: LF; low-frequency component, HF; high-frequency component, nu; indicates normalized units. Significances are *p<0.05 vs. ND-S; †p£0.05 vs. HFD-S and $\$ \mathrm{p}<0.05$ vs. ND-T. (The parametric variables were tested using 2-way ANOVA with Tukey post-test and non-parametric data were determined by Friedman test followed by Mann-Whitney U-tests.)

Figure 2 shows that the linear regression slope between changes in $\mathrm{HR}$ and MBP $(\mathrm{bpm} / \mathrm{mmHg})$ corresponding to reflex bradycardia and reflex tachycardia was greatly attenuated by high-fat diet in sedentary animals $(-0.52 \pm 0.1$ and $1.65 \pm 0.3 \mathrm{bpm} /$ $\mathrm{mmHg}$; respectively). The exercise training improved baroreflex control of HR in HFD-induced obese rats. Consequently, at the end of the 9-week intervention period, the reflex bradycardic sensitivity in HFD-T $(-2.15 \pm 0.2 \mathrm{bpm} / \mathrm{mmHg})$ exceeder that observed in their respective sedentary group $(p<0.001)$. The reflex tachycardic sensitivity was also higher in HFD-T $(2.83$ $\pm 0.2 \mathrm{bpm} / \mathrm{mmHg})$ than HFD-S rats $(\mathrm{p}<0.001)$ and was similar to that observed in ND-S group $(3.04 \pm 0.1 \mathrm{bpm} / \mathrm{mmHg})$. The reflex tachycardia in trained rats fed a normal diet was more pronounced in relation to trained rats fed a high-fat $\operatorname{diet}(\mathrm{p}<0.001)$.

Figure 2. Effects of chronic exercise training on the reflex control of the heart rate in non-anesthetized Wistar rats that were assigned to sedentary normal diet (ND-S, $n=8$ ), sedentary high-fat diet (HFD-S, $n=8$ ), trained normal diet (ND-T, n=8) and trained high-fat diet (HFD-T, $n=8$ ). Baroreflex bradycardic gain and baroreflex tachycardic gain, respectively, induced by phenylephrine and sodium nitroprusside injections. Baroreflex gains were obtained by DHR/DMAP index (bpm/mmHg). Data are mean $\pm \mathrm{SEM}$. ${ }^{*} \mathrm{p}<0.05$ vs. all groups and $\$ \mathrm{p}<0.05 \mathrm{vs}$. ND-T. (Statistical differences were determined by 2-way ANOVA followed by Tukey post-test).

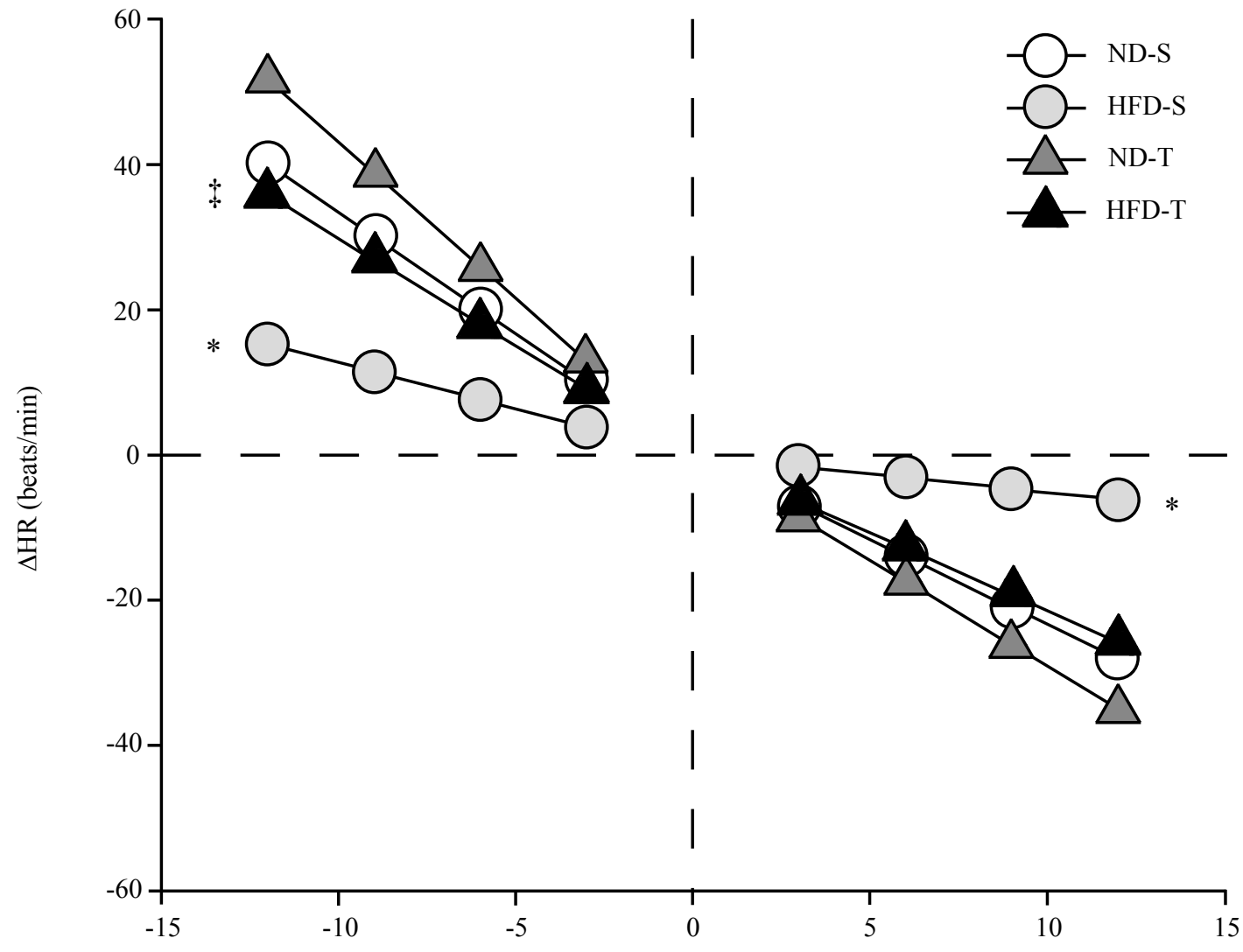

$\triangle \mathrm{MBP}(\mathrm{mmHg})$ 
Compared with all the groups, the HFD-S presented higher values of sympathetic tonus $(\mathrm{p}<0.05)$. The aerobic exercise training could decreased sympathetic tonus in HFD in comparison to their respective sedentary group $(\mathrm{p}<0.05)$. In contrast, both the ND-T and HFD-T groups shows a higher cardiac vagal tonus in comparison to ND-S and HFD-S $(\mathrm{p}<0.05)$ (Fig. 3).

Figure 3. Bar graphs showing sympathetic (ST) and vagal (VT) tone, basal heart rate (HR), intrinsic HR (IHR) and changes (\%) in HR (DHR) after atropine (A) and propranolol (P) injections in conscious Wistar rats that were divided in four treatment groups, respectively, sedentary normal diet (ND-S, $n=8$ ), sedentary high-fat diet (HFD-S, $n=8$ ), trained normal diet (ND-T, $n=8)$ and trained high-fat diet (HFD-T, $n=8$ ). The VT was estimated by the difference between the IHR (double blockade by atropine and propranolol) and the maximal bradycardia achieved after sympathetic blockade with propranolol. The ST was evaluated by the difference between the IHR the highest tachycardia observed after vagal blockade with atropine. Data presented as mean \pm SEM. (Statistical differences were determined by 2-way ANOVA followed by Tukey post-test).

Sympathetic tonus $\square$ Vagal tonus

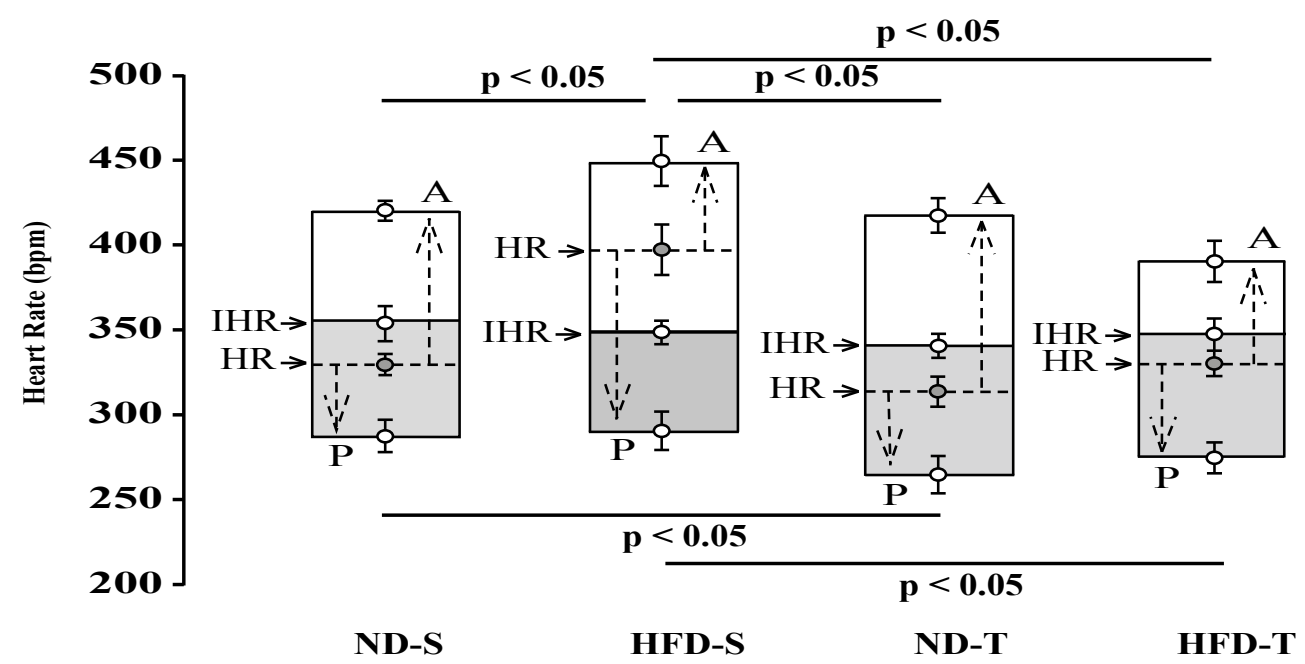

Further analysis showed a significant association between visceral fat, sympathetic nervous activity and BP levels (Fig. 4), demonstrated by correlation of visceral white adipose tissue and LF component of HRV $(r=0.44, p<0.05$; Fig. $4 a)$, white adipose tissue and LF component of SBPV ( $\mathrm{r}=0.56, \mathrm{p}<0.001$; Fig. $4 \mathrm{~b})$, white adipose tissue and LF component of DBPV $(r=0.45$, $\mathrm{p}<0.05$; Fig. $4 \mathrm{c})$. The Figure $4 \mathrm{~d}$ show a correlation between visceral white adipose tissue and $\operatorname{MBP}(\mathrm{r}=0.39, \mathrm{p}<0.05)$. In addition, a strong positive association was also found between sympathetic modulation represented by the LF component of SBPV and MBP $(r=0.81, \mathrm{p}<0.001$; Fig. $4 \mathrm{e})$ and LF component of DBPV and MBP (r=0.76, $\mathrm{p}<0.001$; Fig. 4f).

Figure 4. Correlation coefficient between (a) visceral fat and LF component of heart rate variability (HRV), (b) visceral fat and LF component of systolic blood pressure variability (SBPV), (c) visceral fat and LF component of diastolic blood pressure variability (DBPV), (d) visceral fat and mean blood pressure (MBP), (e) LF component of SBPV and MBP and (f) LF component of DBPV and MBP of non-anesthetized Wistar rats submitted to normal diet plus sedentary activity (ND-S, n=8), high-fat diet plus sedentary activity (HFD-S, n=8), normal diet plus exercise training activity (ND-T, $n=8$ ) and high-fat diet plus exercise training activity (HFD-T, $n=8$ ) treatment during 9 weeks. (Statistical differences were determined by Pearson correlation coefficient).
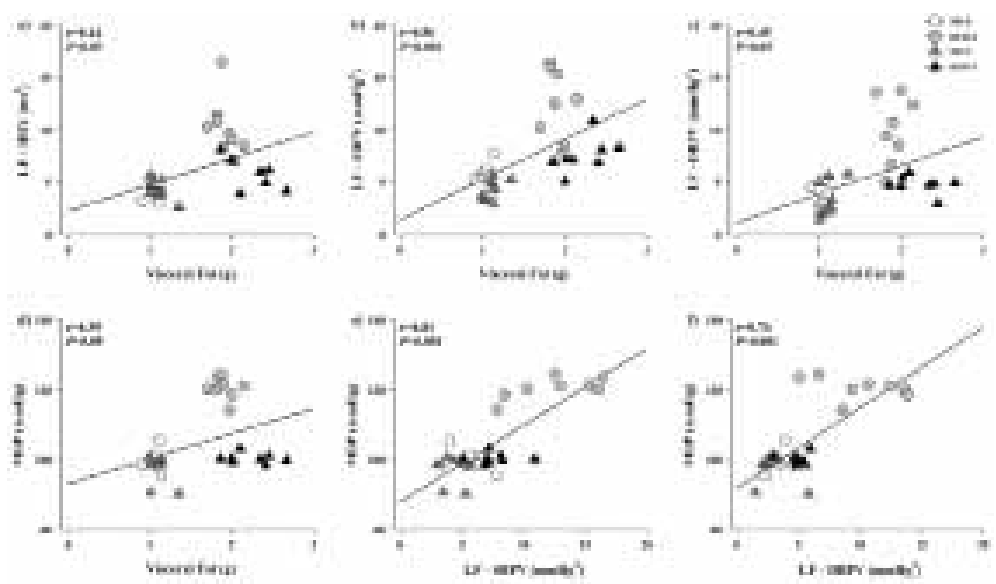


\section{Discussion}

One important finding of our research was that HFD-induced obesity in rats is correlated with autonomic imbalance represented by a sympathetic overactivity, parasympathetic attenuation and reduced response of baroreflex sensitivity, as well as an association between elevated sympathetic activity and higher levels of BP. In addition, conducting a simple aerobic exercise training protocol is effective to reverse these changes.

Previous studies with animals have shown that obesity increases the risk of developing hypertension and other cardiovascular diseases, and excessive sympathetic outflow contributes to the progression of obesity ${ }^{20}$. Feeding rat with a HFD is known to closely mimic many characteristics of human obesity ${ }^{21}$. Corroborating these studies, here we have demonstrated that HFD intake for 9 weeks induced obesity, as evidenced by the increased body weight and adiposity. The above variables indicated the rat model of diet induced obesity was successfully established and are suitable for investigating mechanistic links between obesity and hypertension.

The mechanisms underlying the development of obesityinduced hypertension are multifactorial and complex. Several researches demonstrated that, among neurohumoral, renal, and vascular factors, hyperactivity of the sympathetic nervous system contributes to the etiology of hypertension in obese humans and animal models ${ }^{2}$. In this sense, our data indicate that HFDinduced hypertension is associated with an increase in the LF component of pulse interval, systolic and diastolic BP, as well as an elevated sympathetic tonus to the heart, which are indexes of cardiac sympathovagal balance and vascular sympathetic outflow, respectively ${ }^{22}$.

Strengthening the involvement of sympathetic overactivity in obesity-induced hypertension are the previous findings that: firstly, drugs that block the sympathetic activity lowers BP to a greater extent in obese compared with lean subjects ${ }^{23}$; secondly, chronic treatment with clonidine (centrally acting $\alpha$-agonist hypotensive agent) or $\alpha$ - and $\beta$-adrenergic receptor antagonists reduces SNS activity and prevents the increase in BP in animals fed with HFD and in hypertensive obese patients ${ }^{2}$.

The mechanisms involved in the increased high BP and concomitant sympathetic hyperactivity in high HFD-induced obesity were not addressed in the present study. However, some potential mechanisms may explain these effects observed. Among several neuromodulators involved in activation of the sympathetic modulation, one of the most studied in obesity is leptin, an adipokine secreted by adipocytes proportionally to the degree of adiposity. High levels of leptin could explain, at least in part, the obesity-induced hypertension and sympathetic hyperactivity of humans ${ }^{24}$ and animals models ${ }^{25}$. Thus, leptin would be a link between the excess of adiposity and sympatho excitation ${ }^{26}$.

We showed that exercise training by swimming was able in attenuated the high BP and provoked fat loss, this indicates the therapeutic effect of chronic exercise on the symptoms of obesity. Exercise training has been shown to modulate sympathetic activity and maintain the balance between parasympathetic and sympathetic activity in the long term of many diseases ${ }^{27}$. Based on these studies, the regular physical exercise shows beneficial effects and may be a non-pharmacological strategy for this particular patient group. As previously reviewed ${ }^{28}$, sympathetic hyperactivity is accompanied by the elevated sympathetic outflow to organs such as the heart, kidneys, and blood vessels ${ }^{29}$. Moreover, it can contribute to a further decline of insulin sensitivity, creating a vicious cycle that may promote the development of the metabolic syndrome. Sympathetic nervous system plays a key role in the control of energy balance and body weight ${ }^{29}$, so when the exercise training causes body weight loss and energy expenditure, the excess sympathetic activity is consequentially attenuated.

Another relevant finding of our study was that HFD-induced obesity rat model shown a reduced cardiac vagal tonus, measured directly after cardiac pharmacological parasympathetic blockade. In addition, the evaluation of the heart rate variability (HRV) indices in the present study suggests a decrease in the activity of the parasympathetic autonomic nervous systems in obese animals observed by reduced of the HF component of pulse interval. Previous researches could demonstrate reduced activity in the parasympathetic division of the autonomic nervous system in obese in comparison to lean subjects. Moreover, in other study, have shown the relation between obesity duration and a lowest parasympathetic tone by means of reduced $\mathrm{HRV}^{30}$.

Clinical $^{31}$ as well as experimental studies ${ }^{32}$ have shown that individuals with the greatest reduction in parasympathetic tone have the greatest propensity for sudden cardiac death. Several pathophysiological mechanisms may partially explain the strong association between obesity and the development of cardiovascular disease, including autonomic dysfunction, which, due to a reduced cardiac vagal tone, can alone be an important risk factor for cardiovascular disease and mortality ${ }^{33}$.

The mechanisms by which vagal dysfunction is related to obesity are under debate, and it is uncertain whether this dysfunction is a consequence of obesity or facilitates their development. The hypothalamus is a regulatory center of satiety and of the autonomic nervous system. Therefore, abnormalities in the hypothalamus may cause obesity and autonomic dysfunction ${ }^{34}$. This may explain the alterations observed in the HRV indices.

Our data demonstrate that exercise training may improve the imbalanced parasympathetic activity of HFD-induced obese rats, which was observed to be closely associated with reduction on the fat deposition in these obese rats. In fact, regular exercise is efficient in modify the cardiac autonomic balance by increasing the parasympathetic activity ${ }^{35}$. Exercise training promotes an increase in the capacity of the ANS to meet the demands of the cardiovascular system, thereby increasing HRV. Previous studies have suggested that exercise training promotes adaptations in the cardiac autonomic balance, characterized by a predominance of the parasympathetic autonomic component over the sympathetic component. This statement is based on studies that showed that high frequency oscillations are mostly due to autonomic parasympathetic modulation ${ }^{36}$.

The aerobic exercise training has been broadly used as a non-pharmacological therapy, attenuating the HR and BP levels and decreasing the risk of metabolic and cardiovascular diseases. Resting bradycardia is an excellent hallmark for exercise training adaptation in humans ${ }^{37}$ and animals ${ }^{38}$, thus, its presence in 
the conscious exercise trained demonstrates the effectiveness of the exercise training used in the present study.

The arterial baroreceptor reflex is designed to buffer beat-tobeat fluctuations in BP. Afferent information from arterial baroreceptors is carried to the dorsal medulla and is integrated in the ventrolateral medulla. These areas modulate the cardiovascular function through the ANS $^{39}$. We analyzed in the current study the arterial baroreceptor reflex control in a HFD model in rats. This analysis demonstrated that HFD-induced obese animals had markedly an impairment of arterial baroreflex control of BP. Our interpretation for these data is that the arterial baroreceptor reflex impairment of sympathetic activity is an early marker of cardiovascular dysfunction related to diet-induced obesity and probably a major mechanism leading to sympathoexcitation related to obesity.

Previously, the literature has reported that the sympathoinhibition and sympathoexcitatory effects induced by baroreceptor stimulation and deactivation, respectively, are impaired in different models of hypertension, including obesity ${ }^{40}$. Arterial baroreflex activity is also blunted in obese patients with hypertension, insulin resistance, and early onset diabetes suggesting baroreflex impairment may play a causal role in the sympathoexcitatory state observed in obesity ${ }^{41}$. Physical inactivity, more likely in obese patient, may also be one of the reasons for reduction in baroreflex sensitivity.

Our data showed that 9 weeks of aerobic training restored arterial baroreceptor reflex in HFD-induced obese rats. Whilst exercise training has been shown to improve sympatho inhibition and potentiate baroreflex sensitivity there is a strong evidence that weight loss associated with exercise, in particular abdominal fat loss, is the most important determinant of sympathetic neural adaption to improve hemodynamic and metabolic parameters in adults $^{42}$. It is possible that an improvement in baroreflex function may have something to do with the sympathoinhibition after weight loss. Previous studies have demonstrated that a hypocaloric diet increased the arterial baroreceptor reflex control of sympathetic activity in obese individuals. There is large body of evidence, showing that exercise training attenuates sympathetic overactivity, increases parasympathetic heart rate modulation and enhanced baroreflex sensitivity in several clinics situations ${ }^{43}$.

Some potential mechanisms may explain the increased baroreceptor gain sensitivity after exercise training presently observed. According to the mechanoelastic concept, in the presence of increased vascular compliance, the same pulse pressure can result in increased baroreceptor activation. Because exercise training increases intrinsic aortic compliance in rats and arterial compliance ${ }^{44}$ in humans, we postulate that the improvement in aortic baroreceptor gain sensitivity may be due to an increase in aortic compliance. Although the increase in arterial compliance is an attractive explanation for the enhancement of baroreceptor gain sensitivity produced by exercise training, it appears to apply to normotensive but not hypertensive rats.

Both the magnitude and frequency of shear stress on the endothelial cells during exercise increase the release of endothelial factors and/or the sensitivity of endothelial cells, which in turn enhances baroreceptor ending activity. In fact, some authors reported that exercise training increases the vasodilatory response to acetylcholine in hypertensive rats ${ }^{45}$. The increase in aortic baroreceptor gain sensitivity may be also explained by a reduction nerve sympathetic nerve activity. Exercise training reduces sympathetic activity and the spillover of catecholamine in humans and reduces the renal sympathetic nerve activity in rats. These changes in sympathetic outflow could modify the distensibility of the sinus area and, in consequence, improve afferent baroreceptor discharge ${ }^{46}$.

Epidemiological and clinical studies have been shown that exercise training is an important approach in preventing of many cardio metabolic diseases and exerts important adaptations in skeletal muscle and adipose tissue metabolism ${ }^{47}$. In this study, we found that regular physical exercise did not change any parameters of body weight, white adipose tissue, BPV and HRV between both sedentary and trained groups that received normal diet. A possible explanation for these results is that administration of the normal diet was not sufficient to cause deleterious changes of these parameters in rats. On the other hand, the exercise training was able to decrease basal heart rate in ND-T group compared to ND-S, as well as increased cardiac vagal tonus. Thus, long-term exercise training used in our study had positive effects on cardiovascular autonomic parameters.

Lifestyle changes, including regular physical activity, have been reported to be more effective in preventing diseases than drug therapy ${ }^{47}$. It is evident that the benefits of exercise training result from a combination of effects on various organs. Previous studies showed that animal models with low aerobic treadmill running capacity (low-intrinsic running capacity) in the absence of exercise training express a number of characteristics common to metabolic disease phenotypes, such as increased body mass and adiposity, hyperinsulinemia, and impaired glucose tolerance ${ }^{48,49}$. In contrast, rats of high aerobic treadmill running capacity (high-intrinsic running capacity) live 6-8 month longer $^{50}$ and present with superior metabolic health characterized by resistance to weight gain and an increased capacity for the uptake and oxidation of glucose and fat ${ }^{48,49}$.

\section{Conclusions}

In summary, we have demonstrated that daily administration of fat diet in rats leads to obesity and cause hypertension and cardiovascular autonomic dysfunction. The elevated fatness levels are associated with sympathetic activation as well as an association between sympathetic hyperactivity and high blood pressure. Nevertheless, the non-pharmacological treatment through exercise training is effective in reversing these harmful changes, and may be considered fundamental to protect of the cardiovascular risk factors acquired with excess body fat.

\section{Reference}

1. Benatti FB, Pedersen BK. Exercise as an anti-infammatory therapy for rheumatic diseases-myokine regulation. Nat Rev Rheumatol. 2015;11(22):86-97. 
2. Hall JE, da Silva AA, do Carmo JM, Dubinion J, Hamza S, Munusamy S, et al. Obesity-induced hypertension: role of sympathetic nervous system, leptin, and melanocortins. J Biol Chem. 2010;285(23):17271-6.

3. Hyatt Sachs H, Rohrer H, Zigmond RE. The conditioning lesion effect on sympathetic neurite outgrowth is dependent on gp130 cytokines. Exp. Neurol. 2010;223(2):516-22.

4. Bedi M, Khullar S, Varshney V P. Assessment of Autonomic Function Activity in Obese Children. Vascular Disease Prevention. 2009;6(2):139-41.

5. Rajalakshmi R, VijayaVageesh Y, Nataraj SM, MuraliDhar SC.. Heart rate variability in Indian obese young adults. Pak. J. Physiol. 2012;8(1):39-44.

6. Mitrani P, Srinivasan M, Dodds C, Patel MS. Autonomic involvement in the permanent metabolic programming of hyperinsulinemia in the high-carbohydrate rat model. Am. J. Physiol. Endocrinol. Metab. 2007;292(5):1364-77.

7. Laederach-Hofmann K, Mussgay L, Rúddel H. Autonomic cardiovascular regulation in obesity. J Endocrinol. 2000;164(1):59-66.

8. Sowers JR. Diabetes mellitus and vascular disease. Hypertension. 2013;61(5):943-47.

9. Afonso RA, Lautt WW, Schafer J, Legare DJ, Oliveira AG, Macedo MP. High-fat diet results in postprandial insulin resistance that involves parasympathetic dysfunction. Br. J. Nutr. 2010;104(10):1450-59.

10. Chen GY, Hsiao TJ, Lo HM, Kuo CD. Abdominal obesity is associated with autonomic nervous derangement in healthy Asian obese subjects. Clin Nutr. 2008;27(2):212-7.

11. Li Y. Association between resting heart rate and cardiovascular mortality: evidence from a meta-analysis of prospective studies. International Journal of Clinical and Experimental Medicine. 2015;8(9):15329-39.

12. Nagai N, Moritani T. Effect of physical activity on autonomic nervous system function in lean and obese children. Int. J. Obes. Relat. Metab. Disord. 2004;28(1):27-33.

13. Rennie KL, McCarthy N, Yazdgerdi S, Marmot M, Brunner E. Association of the metabolic syndrome with both vigorous and moderate physical activity. Int. J. Epidemiol. 2003;32(4):600-6.

14. Estadella D, Oyama LM, Dâmaso AR, Ribeiro EB, Oller Do Nascimento CM. Effect of palatable hyperlipidic diet on lipid metabolism of sedentary 39 and exercised rats. Nutrition. 2004;20(2):218-24.

15. Seo TB, Han LS, Yoon JH, Hong KE, Yoon SJ, Namgung UK. Involvement of $\mathrm{Cdc} 2$ in axonal regeneration enhanced by exercise training in rats. Med. Sci. Sports Exerc. 2006;38(7):1267-76.

16. Gobatto CA, Mello MA, Sibuya CY, Azevedo JR, Santos LA, Kokubun E. Maximal lactate steady state in rats submitted to swimming exercise. Comp. Biochem. Physiol. A. Mol. Integr. Physiol. 2001;130(1):21-7.

17. Malliani A, Pagani M, Lombardi F, Cerutti S. Cardiovascular neural regulation explored in the frequency domain. Circulation. 1991;84(2):482-92.

18. Tfescnaspe. Task Force of the European Society of Cardiology and the North American Society of Pacing and Electrophysiology. Heart rate variability: standards of measurement, physiological interpretation and clinical use. Circulation. 1996;93(5):1043-65.
19. Rubini R, Porta A, Baselli G, Cerutti S, Paro M. Power spectrum analysis of cardiovascular variability monitored by telemetry in conscious unrestrained rats. J. Auton. Nerv. Syst. 1993;45(3):181-90.

20. Chaar LJ, Coelho A, Silva NM, Festuccia WL, Antunes VR. High-fat diet-induced hypertension and autonomic imbalance are associated with an upregulation of CART in the dorsomedial hypothalamus of mice. Physiological Reports. 2016;4(11):1-14.

21. Northcott CA, Fink GD, Garver H, Haywood JR, LaimonThomson EL, Mcclain JL, et al. The development of hypertension and hyperaldosteronism in a rodent model of life-long obesity. Endocrinology. 2012;153(4):1764-73.

22. Thireau J, Zhang BL, Poisson D, Babuty D. Heart rate variability in mice: a theoretical and practical guide. Exp. Physiol. 2008;93(1):83-94.

23. D'Angelo G, Mintz JD, Tidwell JE, Schreihofer AM, Pollock DM, Stepp DW. Exaggerated cardiovascular stress responses and impaired-adrenergic-mediated pressor recovery in obese Zucker rats. Hypertension. 2006;48(6):1109-15.

24. Eikelis N, Schlaich M, Aggarwal A, Kaye D, Esler M. Interactions between leptin and the human sympathetic nervous system. Hypertension. 2003;41(5):1072-9.

25. Simonds SE, Pryor JT, Ravussin E, Greenway FL, Dileone R, Allen AM, et al. Leptin mediates the increase in blood pressure associated with obesity. Cell. 2014;159(6):1404-16.

26. Rahmouni K, Morgan DA, Morgan GM, Mark AL, Haynes WG. Role of selective leptin resistance in diet-induced obesity hypertension. Diabetes. 2005;54(7):2012-8.

27. Broderick TL, Wang D, Jankowski M, Gutkowska J. Unexpected effects of voluntary exercise training on natriuretic peptide and receptor mRNA expression in the ob/ob mouse heart. Regul. Pept. 2014;188(2):52-9.

28. Davy KP, Orr JS. Sympathetic nervous system behavior in human obesity. Neurosci Biobehav Rev. 2009;33(2):116-24.

29. Messina G, De Luca V, Viggiano A, Ascione A, Iannaccone T, Chieffi S, Monda M. Autonomic nervous system in the control of energy balance and body weight: personal contributions. Neurol. Res. Int. 2013;639280.

30. Rabbia F, Silke B, Conterno A, Grosso T, DeVito B, Rabbone I, et al. Assessment of cardiac autonomic modulation during adolescent obesity. Obes Res. 2003;11(4):541-8.

31. Kleiger RE, Miller JP, Bigger JT Jr, Moss AJ. Decreased heart rate variability and its association with increased mortality after acute myocardial infarction. Am. J. Cardiol. 1987;59(4):256-62.

32. Collins MN, Billman GH. Autonomic response to coronary occlusion in animals susceptible to ventricular fibrillation. Am. J. Physiol. 1989; 257(6 Pt 2):1886-94.

33. Lauer MS. Autonomic function and prognosis. Cleve Clin. J. Med. 2009;76(1):18-22.

34. Tonhajzerova I, Javorka M, Trunkvalterova Z, Chroma O, Javorkova J, Lazarova Z, et al. Cardio-respiratory interaction and autonomic dysfunction in obesity. J. Physiol. Pharmac. 2008;59(Suppl 6):709-18.

35. Abreu SB, Lenhard A, Mehanna A, de Souza HC, Correa FM, Hasser EM, et al. Role of paraventricular nucleus in exercise training-induced autonomic modulation in conscious rats. Auton Neurosci. 2009;148(1-2):28-35. 
36. Souza HC, De Araujo JE, Martins-Pinge MC, Cozza IC, MartinsDias DP. Nitric oxide synthesis blockade reduced the baroreflex sensitivity in trained rats. Auton. Neurosci. 2009;150(1-2):38-44.

37. Sugawara J, Murakami H, Maeda S, Kuno S, Matsuda M. Changes in post-exercise vagal reactivation with exercise training and detraining in young men. Eur. J. Appl. Physiol. 2001;85(3-4):259-63.

38. Schaefer ME, Allert JA, Adams HR, Laughlin MH. Adrenergic responsiveness and intrinsic sinoatrial automaticity of exercisetrained rats. Med. Sci. Sports Exerc. 1992;24(8):887-94.

39. Dampney RA. Functional organization of central pathways regulating the cardiovascular system. Physiol. Rev. 1994;74(2):323-64.

40. Huber DA, Schreihofer AM. Attenuated baroreflex control of sympathetic nerve activity in obese Zucker rats by central mechanisms. J. Physiol. 2010;588(Pt 9):1515-25.

41. Straznicky NE, Lambert EA, Lambert GW, Masuo K, Esler MD, Nestel PJ. Effects of dietary weight loss on sympathetic activity and cardiac risk factors associated with the metabolic syndrome. J. Clin. Endocrinol. Metab. 2005;90(11):5998-6005.

42. Patel KP, Salgado HC, Liu X, Zheng H. Exercise training normalizes the blunted central componente of the baroreflex in rats with heart failure: role of the PVN. Am. J. Physiol. Heart. Circ. Physyol. 2013;305(2):173-81.

43. Liu, JL, Kulakofsky J, Zucker IH. Exercise training enhances baroreflex control of heart rate by a vagal mechanism in rabbits with heart failure. J. Appl. Physiol. 2002; 92 (6): 2403-2408.

44. Kingwell BA, Cameron JD, Gillies KJ, Jennings GL, Dart AM. Arterial compliance may influence baroreflex function in athletes and hypertensives. Am. J. Physiol. 1995; 268 (1 Pt 2): H411-H418.

45. Yen MH, Yang JH, Sheu JR, Lee YM, Ding YA. Chronic exercise enhances endothelium-mediated dilation in spontaneously hypertensive rats. Life Sci. 1995; 57 (24): 2205-2213.

46. Seagard JL, Hopp FA, Kampine JP. Effect of sympathetic sensitization of baroreceptors on renal nerve activity. Am. J. Physiol. 1987; 252 (2 Pt 2): R328-R335.
47. Gordecke JH, Micklesfield LK. The effect of exercise on obesity, body fat distribution and risk for type 2 diabetes. Med Sport Sci. 2014; 60, 82-93.

48. Noland RC, Thyfault JP, Henes ST, Whitfield BR, Woodlief TL, Evans JR, et al. Artificial selection for high-capacity endurance running is protective against high-fat diet-induced insulin resistance. Am J Physiol Endocrinol Metab. 2007; 293 (1): E31-E41.

49. Stephenson EJ, Stepto NK, Koch LG, Britton SL, Hawley JA. Divergent skeletal muscle respiratory capacities in rats artificially selected for high and low running ability: a role for Nor1? J Appl Physiol. 2012; 113 (9): 1403-1402.

50. Koch LG, Kemi OJ, Qi N, Leng SX, Bijma P, Gilligan LJ, et al. Intrinsic aerobic capacity sets a divide for aging and longevity. Circ Res. 2011; 109 (10): 1162-1172.

\section{Acknowledgements}

We acknowledge the Federal University of Triângulo Mineiro that made this study possible. We also thank Izabella Cristina Ferreira and Tatiane Mocellin Bolelli for contributions to this study.

\section{Corresponding author}

*Octávio Barbosa Neto, Ph.D.

Human Performance and Sport Research Group, Department of Sport Sciences, Federal University of Triangulo Mineiro. Av. Getúlio Guaritá, 159, Postcode (CEP): 38025-440, Uberaba, Brazil

Email: octavio@ef.uftm.edu.br

Manuscript received on September 1, 2017

Manuscript accepted on November 4, 2017

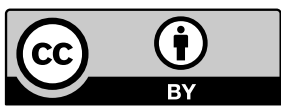

Motriz. The Journal of Physical Education. UNESP. Rio Claro, SP, Brazil - eISSN: 1980-6574 - under a license Creative Commons - Version 3.0 